\title{
Exosomes from adipose mesenchymal stem cells overexpressing Stanniocalcin-1 promote reendothelialization after carotid endarterium mechanical injury
}

Kun Liu ( $\nabla$ dr.liukun@hotmail.com )

Shanghai 9th Peoples Hospital Affiliated to Shanghai Jiaotong University School of Medicine Huihua Shi

Shanghai 9th Peoples Hospital Affiliated to Shanghai Jiaotong University School of Medicine Zhiyou Peng

Shanghai 9th Peoples Hospital Affiliated to Shanghai Jiaotong University School of Medicine Xiaoyu Wu

Shanghai 9th Peoples Hospital Affiliated to Shanghai Jiaotong University School of Medicine

\section{Weimin Li}

Shanghai 9th Peoples Hospital Affiliated to Shanghai Jiaotong University School of Medicine

\section{Xinwu Lu}

Shanghai 9th Peoples Hospital Affiliated to Shanghai Jiaotong University School of Medicine

\section{Research Article}

Keywords: ADSC, Exosome, Stanniocalcin-1, NLRP3 inflammasome, reendothelialization

Posted Date: February 24th, 2021

DOl: https://doi.org/10.21203/rs.3.rs-250063/v1

License: (c) (1) This work is licensed under a Creative Commons Attribution 4.0 International License. Read Full License

Version of Record: A version of this preprint was published at Stem Cell Reviews and Reports on May 12th, 2021. See the published version at https://doi.org/10.1007/s12015-021-10180-4. 


\section{Abstract}

Stanniocalcin-1 (STC-1) is a secreted glycoprotein that participates in the regulation of inflammation, apoptosis, and necrosis. We investigated the reendothelialization effect of exosomes from adipose stem cells (ADSC) overexpressing STC-1 on injured carotid endarterium. ADSCs were transfected with lentivirus vectors containing pre-STC-1. PHK-26 as molecular probe was used to track the exosomes engulfed by mice arterial endothelial cells (MAEC). The role of STC-1-ADSC-Exosome (S-ADSC-Exo) in MAECs was verified through scratch test and tube forming. Expressions of STC-1 and NLRP3 inflammasome were detected by western blot and quantitative reverse transcription polymerase chain reaction.

Reendothelialization effect was inhibited by the antagonist of siRNA targeting STC-1. Carotid endarterium mechanical injury was induced by insertion with a guidewire into the common carotid artery lumen. Carotid arteries were harvested for histological examination, immunofluorescence staining, and Evan's blue staining. Transfection of STC-1 significantly enhanced STC-1 levels in ADSCs, their exosomes, and MAECs. Compared with the control group and the ADSC-Exo group, STC-1 enriched exosomes markedly inhibited the expressions of NLRP3, Caspase-1, and IL-1 $\beta$ in MAECs, exhibited good lateral migration capacity, and promoted angiogenesis. Administration of siRNA targeting STC-1 completely abolished down-regulation of NLRP3, Caspase-1, and IL-1 $\beta$ by STC-1 and inhibited effects of S-ADSC-Exo on lateral migration and angiogenesis. In vivo administration of S-ADSC-Exo had reendothelialization effect on post-injury carotid endarterium as evidenced by thinner arterial wall, low-expressed NLRP3 inflammasome, and more living endothelial cells. The reendothelialization effect of exosomes from ADSCs on post-injury carotid endarterium can be enhanced by genetic modification to contain elevated STC-1.

\section{Introduction}

Approximately 18-25\% ischemic strokes are caused by extracranial carotid atherosclerotic stenosis[1]. For patients at high risk of surgical complications, combination carotid angioplasty with stent implantation has become an accepted alternative to endarterectomy[2]. Despite refinements of interventional technique, the mechanical injury to carotid endarterium is still unavoidable completely and subsequent inflammatory restenosis is not conducive to ensure the long-term patency[3]. Post-injury restenosis just like wound-healing comprises complex pathophysiological mechanisms consisting of inflammation, proliferation, and migration followed by remodeling of arterial wall[4]. Persistent and exaggerated inflammation triggers the release of numbers of cytokines and growth factors, resulting in hyperplasia of neointima and vascular restenosis[5]. Reendothelialization is a crucial repair process that endothelial cells rapidly attempt to cover the denuded area by migrating over distance and increasing in number in response to any injury to endothelium[6]. Delayed reendothelialization and impaired endothelial function after mechanical injury are linked to stent thrombosis. Therefore, inhibition of inflammation, restoration of endothelial cell function to accelerate reendothelialization process are effective approaches to prevent and treat restenosis after endovascular treatment. 
Exosomes carry a wide range of bioactive molecules, such as DNAs, mRNAs, microRNAs, cytoskeletal elements, protease, signaling molecules and play important roles in intracellular information communication[7, 8]. Bioactive molecules can be transported to target cells under physiologic and pathologic conditions, which induce functional and expressional changes[8]. Recently, with regard to the role in the process of inflammation, exosomes derived from different cell types have drawn much interest. Via exosomes, IL-4 modified macrophages foster M2 polarization and inhibit inflammation to retard atherosclerosis through transferring their regulatory microRNAs to target cells[9]. Exosomes generated from cardiomyocytes and endothelial cells followed acute myocardial infarction are taken up by macrophages and regulate local inflammation to attenuate ischemic injury[10]. Mesenchymal stem cells (MSC) are multipotential stem cells that can promote immunomodulation, angiogenesis, matrix remodeling in the injured vessels by secreting factors to activate the signaling pathways involved in vascular repair. The powerful paracrine capacity of MSCs, not their differentiation potential, is the principal mechanisms of their repair action[11]. Local transplantation of adipose-derived mesenchymal stem cells around arteriovenous fistula alleviates restenosis and restores patency after PTA in mice[12]. MSCs have been reported to exert their powerful immunomodulatory and anti-inflammatory effects in the pathological process of atherosclerosis[13]. However, the microenvironment of target tissue may affect ADSCs survival and migration, which leads to poor long-term prognosis[14]. Compared with other cell types, MSCs are more likely to secrete a great quantity of exosomes, and exosomes can overcome the above deficiencies because of their low immunogenicity[15]. Exosomes can protect their contents against the interference of surrounding microenvironment and effectively enhance the intercellular information communication mimicking the molecular biological functions of MSCs[8]. Umbilical cord mesenchymal stem cell-derived exosomes have been indicated to attenuate TNF-a induced inflammation in endothelial cells[16]. Mesenchymal stromal cell-derived exosomes are shown to ameliorate diabetic peripheral neuropathy by suppression of proinflammatory genes[17]. With the development of exosome research, it has been found that the function of exosomes can be enhanced by genetic modification of stem cells to load target proteins[18].

Interleukin (IL) - $1 \beta$ has been reported to participate in the chronic inflammation and restenosis after endovascular treatment[12, 19]. As an important component of innate immunity, NLRP3 inflammasome consisting of NLRP3 receptor protein, apoptosis-associated speck-link protein containing a CARD (ASC), and Caspase- 1 has recently emerged as a protein complex for inducing the release of IL-1 $\beta$ and IL-18[20]. ROS are key molecule signal for activating NLRP3[21], and proved to be triggered after stent implantation and PTA[22]. STC-1 is a kind of glycoprotein directly act on mitochondria and then inhibit ROS through uncoupling[23].Thus, it is plausible that the axis of STC-1-NLRP3- IL-1 $\beta$ may be the new target for protecting endothelial cells against inflammation induced by mechanical injury and promoting reendothelialization. In the current study, we delivered STC-1 to endothelial cells by exosomes from ADSCs that were genetically modified to overexpress STC-1. We highlight the reendothelialization effect of STC-1-modified exosomes on post-injury carotid endarterium in the setting of inflammation caused by mechanical injury. 


\section{Materials And Methods}

\section{Animals}

Male, 6-8 weeks C57/BL mice were enrolled in the present study.

\section{Isolation, culture, identification and transfection of ADSCs}

The primary ADSCs were extracted from the adipose tissue of the inguinal area of C57/BL mice. The harvested adipose tissues were digested with NB4 collagenase (Nordmark, Uetersen, German) in low glucose (1000 mg mL ${ }^{-1}$ ) Dulbecco's Modified Eagle's Medium (DMEM) supplemented with 10\% fetal bovine serum (FBS, Gibco, Waltham, U.S.A). After 60 min digesting and shaking (220 rpm) at room temperature, the cells were resuspended and seeded on dishes, and used at passage 3 . To identify the phenotype of ADSCs, the specific surface markers were determined by flow cytometry analysis (Beckman Coulter, Fullerton, CA). The mouse antibodies against CD29, CD31, CD34, and CD44 were conjugated with FITC. ADSCs were incubated with the above antibodies in $100 \mathrm{~mL}$ FACS buffer (Sigma, San Francisco, U.S.A). The labeled cells were washed three times with $1.5 \mathrm{~mL}$ FACS buffer and fixed with cytofix (BD Pharminogen, San Francisco, U.S.A). The Beckman Coulter flow cytometer and the FlowJo software (Version 10.0) were used to analyze data. Mice STC-1 lentivirus gene transfer vectors were constructed by Genechem (Shanghai, China). 1'106 ADSCs were seeded in $10 \mathrm{~mL}$ plate overnight and were subsequently transfected with 200nM STC-1 vector at a multiplicity of infection of 10 for 24 hours. After that, the envelope suspension was transferred to normal culture medium, and the transfected ADSCs were cultured for 48 hours.

\section{Exosomes extraction and identification}

At 48 hours after transfection, the exosomes were isolated at $4^{\circ} \mathrm{C}$ according to the method previously described[24]. Firstly, the culture medium of ADSCs was filtered to remove large debris and floating cells. Secondly, small debris was removed by centrifugation at 10,000'g for $30 \mathrm{~min}$. Lastly, the remaining supernatants were further centrifugated at $10,000^{\prime} \mathrm{g}$ for $3 \mathrm{~h}$. The precipitate was resuspended in phosphate-buffered saline (PBS). The morphologic characteristics of exosome were observed using transmission electron microscopy (TEM). Expression of CD9, CD63 and CD81, as markers of exosomes, were confirmed by Western blot.

\section{Tracking of exosomes engulfed by MAECs}

MAECs were purchased from Daixuan Biosciences Inc (Shanghai, China) and cultured in high glucose (4500 mg mL $\mathrm{m}^{-1}$ ) DMEM supplemented with 10\% fetal bovine serum and 1\% penicillin-streptomycin. MAECs were cocultured with PHK26 labelled exosomes for $24 \mathrm{~h}$. The exosomes engulfed by MAECs were identified by fluorescence microscopy. DAPI was used to stain nucleus, and Phalloidin was used to stain cytoskeleton. 
NLRP3 inflammasome was activated according to the method previously described[23]. MAECs (1 $' 10^{5} /$ well) were seed in 6-well plates. The culture medium was added with LPS (Ultra-pure InvivoGen, San Diego, CA) at the concentration of $2 \mathrm{mg} \mathrm{mL}^{-1}$ in high glucose DMEM for 6 hours. After washing with PBS three times, the MAECs were incubated with $5 \mathrm{mM}$ ATP (InvivoGen, San Diego, CA) for $45 \mathrm{~min}$. After that, the MAECs were washed with PBS for three times and cultured in high glucose DMEM. Scrapping with a pipette tip was conducted in the upper 3 wells of 6 -well plate, with the lower 3 wells receiving no procedure as control. 24 hours later, the cells were collected to determine the expression of NLRP3 inflammasome.

\section{STC-1 Silencing}

To silence STC-1, small interfering RNA (siRNA) targeting STC-1 was purchased from Thermo Fisher Scientific (USA). MAECs $\left(1^{\prime} 10^{5} /\right.$ well) were seed in 6-well plates until reaching $70-80 \%$ confluence. The cells were washed with PBS and transfected with siRNA-STC-1, or nontargeting siRNA using lipofectamine 3000 according manufacturer's protocol. After 6 hours incubation, the medium was replaced with fresh DMEM for 48 hours incubation. These MAECs were used to perform scratch test and tube forming experiment. RT-qPCR and western blot were performed to evaluate the knockdown efficiency between the S-ADSC-Exo group and the siRNA-STC-1 group.

\section{Scratch test and Tube forming experiment}

The lateral migration capacity of MAECs was assessed by scratch test. $1^{\prime} 10^{5}$ MAECs were seed in 6-well plates and scraped by a pipette tip to generate uniform wounds when reached at approximately $90 \%$ confluent. Each well was washed three times with PBS to remove floating cells, and then the MAECs were cultured in medium with no serum. The blank area at the time intervals ( $0 \mathrm{~h}, 6 \mathrm{~h}, 12 \mathrm{~h}$, and $24 \mathrm{~h}$ ) were observed under inverted microscope and calculated using Image-Pro Plus 6.0 software.

$10 \mathrm{~mL}$ of Matrigel per well was evenly placed on the angiogenic slide (Ibidi, Germen). After that, the slide was placed in an incubator for 2 hours to solidify the Matrigel. About $1^{\prime} 10^{4}$ MAECs were seeded on per well and observed under inverted microscope after 4-6 hours.

\section{Animal model of carotid endarterium wire-injury and tail vein injection}

To avoid bias that may be caused by surgical procedure, the mice were randomly divided. All operations were conducted by one researcher to ensure the consistency. After administering anesthesia (pentobarbital sodium, $0.5 \mathrm{mg} \mathrm{g}^{-1}$ ), the mice were fixed on a heating plate maintained at $37^{\circ} \mathrm{C}$. A middle neck incision was performed, the left common carotid artery and its branches were skeletonized. After dissection, mechanical injury to carotid endarterium was induced by insertion with an 0.014 " guide-wire into the left common carotid artery. At the end of procedure, the guide-wire was removed, the proximal and distal sutures were tied off gently. The administration of exosomes was performed through tail vein injection. The procedure is shown in Fig. S1. 


\section{Experimental protocol}

Part I. To evaluate the reendothelialization effect of S-ADSC-Exo.

In vitro. The NLRP3 inflammasome was activated by addition of LPS + ATP according to the method as described above. The scratch test: $₫$ Control group: PBS $(2 \mathrm{~mL}), \triangle A D S C$-Exo group: ADSC-Exo $(2 \mathrm{~mL}, 2 \mathrm{mg}$ $\left.\mathrm{mL}^{-1}\right)$, and $\triangle \mathrm{S}$-ADSC-Exo group: S-ADSC-Exo $\left(2 \mathrm{~mL}, 2 \mathrm{mg} \mathrm{mL}^{-1}\right)$ was respectively added to the 6-well plate cultured with MAECs $\left(1^{\prime} 10^{5} /\right.$ well) after being scraped by a pipette tip. The tube forming experiment: $\mathbb{\nabla}$ Control group: PBS $(2 \mathrm{~mL}), \triangle A D S C$-Exo group: ADSC-Exo $\left(2 \mathrm{~mL}, 2 \mathrm{mg} \mathrm{mL}^{-1}\right)$, and $\otimes \mathrm{S}$-ADSC-Exo group: SADSC-Exo $\left(2 \mathrm{~mL}, 2 \mathrm{mg} \mathrm{mL}^{-1}\right)$ was respectively added to the angiogenic slide cultured with MAECs $\left(1^{\prime} 10^{4}\right.$ /well). In a parallel series of experiments, MAECs were collected from the 3 groups 24 hours after being scraped to determine the expressions of NLRP3 inflammasome.

In vivo. $\otimes$ Control group $(\mathrm{n}=10)$ : PBS (100mL), $\triangle$ ADSC-Exo group $(\mathrm{n}=10)$ : ADSC-Exo $\left(100 \mathrm{~mL}, 2 \mathrm{mg} \mathrm{mL}^{-1}\right)$, and $\triangle$ S-ADSC-Exo group $(n=10)$ : S-ADSC-Exo $\left(100 \mathrm{~mL}, 2 \mathrm{mg} \mathrm{mL}^{-1}\right)$ was respectively injected through tail vein 1 hour after left common carotid artery wire-injury. All the mice were sacrificed 14 days after operation, and left common carotid arteries were harvested for histological examination, immunofluorescence staining, and Evan's blue staining. In a parallel series of experiments, the left common carotid arteries were additionally harvested from the 3 groups $(n=5)$ to determine the expressions of NLRP3 inflammasome.

Part II. To evaluate the antagonist of siRNA-STC-1.

In vitro. The NLRP3 inflammasome was activated by addition of LPS + ATP according to the method as described above. The scratch test: $₫$ Control group: PBS $(2 \mathrm{~mL}), \nabla$ S-ADSC-Exo group: S-ADSC-Exo $(2 \mathrm{~mL}, 2$

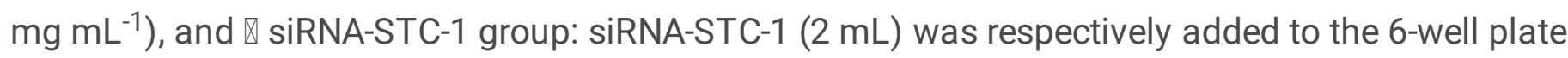

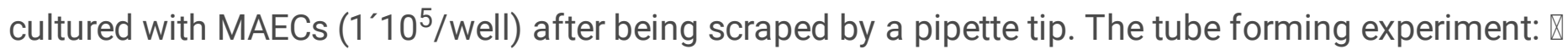

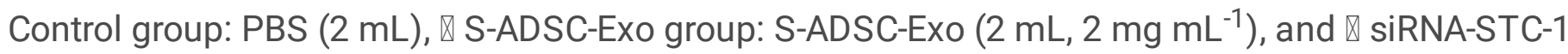
group: siRNA-STC-1 (2 mL) was respectively added to the angiogenic slide cultured with MAECs $\left(1^{\prime} 10^{4}\right.$ /well). In a parallel series of experiments, MAECs were collected from the 3 groups 24 hours after being scraped to determine the expressions of NLRP3 inflammasome.

\section{Enzyme-Linked Immunosorbent Assay}

Contents of ROS were measured using enzyme-linked immunosorbent assay kits according to the manufacturer's instructions (Thermo Fisher, USA).

\section{Histological examination}

On the $14^{\text {th }}$ day after procedure, the post-injury carotid arteries were harvested for histological analysis. Paraffin-embedded sections ( $4 \mathrm{~mm}$ ) of left common carotid arteries were stained with hematoxylin-eosin $(\mathrm{HE})$ dye to determine the thickness of arterial wall. Immunofluorescence staining of the left common 
carotid arteries was performed to determine the expression of NLRP3 inflammasome. To determine the reendothelialization effect on post-injury carotid endarterium, $1 \%$ Even's blue dye was infused into the carotid arteries to delineate the injured area from reendothelialized area.

\section{Quantitative real-time polymerase chain reaction}

Total RNA of ADSCs, exosomes, and MAECs was isolated with Trizol reagent (Invitrogen, Carlsbad, Calif) according to the manufacturer's instruction. Quantitative real-time polymerase chain reaction was conducted according to a standard protocol with the Step One Plus system (Applied Biosystems, Foster City, Calif). GAPDH was used as internal control. Expressions were determined by $2^{-\triangle \triangle C T}$ method.

\section{Western blot}

STC-1 in ADSCs after transfection, CD9, CD63, CD81, and STC-1 in their exosomes and NLRP3, Caspase1 , and IL-1 $\beta$ in MAECs were determined by sodium dodecyl sulfate polyacrylamide gel electrophoresis and immunoblotted with anti-STC-1, anti-CD9, anti-CD63, anti-CD81, anti-NLRP3, anti-Caspase-1, and antiIL-1 $\beta$ antibodies (Abcam, Cambridge, Mass). Expressions of these proteins were analyzed with NIH Image (Research Services Branch, National Institutes of Health, Bethesda, Md) and quantified as relative folds to the control group after normalization with GAPDH.

\section{Statistical analysis}

Parametric values were analyzed by one-way analysis of variance followed by Bonferroni correction for post hoc testing. $P<.05$ was considered statistically significant. Statistical analysis was performed with SPSS, version 25.0 (IBM-SPSS Inc, Armonk, NY).

\section{Results}

\section{Characterization of ADSCs and exosomes}

ADSCs at passage 3 (Fig. S2 a) were used in the following experiments. Flow cytometry analysis showed that specific surface antigens of stem cell (CD29 and CD44) were strongly positive, while the specific surface antigens of hematopoietic cell (CD31 and CD34) were negative (Fig. S2 b). Exosomes derived from ADSCs were membrane vesicles (Fig. S2 c) and there is no difference in morphology between ADSCExo and S-ADSC-Exo. The surface markers CD9, CD63, and CD81 of exosomes were confirmed by western blot (Fig. S2 d).

\section{ADSC-Exosomes engulfed by MAECs and expressions of STC-1 after transfection}

The PHK26 labeled exosomes were engulfed and detected in MAECs (Fig. 1 a). Fluorescence microscopy showed that PHK-26 labeled exosomes were stained (red), with nuclear staining with DAPI (blue), and cytoskeleton staining with Phalloidin (green). Representative western blot pictures of STC-1 in ADSCs, their exosomes, and MAECs engulfing ADSC-Exo are shown in Fig. 1 b-d. Densitometric analysis revealed 
that compared with the un-transfected groups, transfection of STC-1 markedly enhanced the expressions of STC-1 in ADSCs, their exosomes, and MAECs engulfing ADSC-Exo (Fig. $1 \mathrm{e}-\mathrm{g})(P<.05)$.

\section{Reendothelialization effect of S-ADSC-Exo in vitro}

After activation of NLRP3 inflammasome, the scratch test was performed to assess the lateral migration capacity of MAECs. Compared with the control group and the ADSC-Exo group, excellent lateral migration capacity was observed in the S-ADSC-Exo group (Fig. 2 a). Blank area calculation showed that the SADSC-Exo group had the fastest migration speed at time intervals ( 6 h, 12 h, 24 h) (Fig. 2 c). Meanwhile, compared with the control group, recovery after scratching was faster in the ADSC-Exo group.

To assess the angiogenesis effect of S-ADSC-Exo on MAECs, tube forming experiment was performed. The results revealed that tube-forming ability of MAECs was significantly improved in the group incubated with S-ADSC-Exo. As shown in Fig. 2 b, d, tube density in the S-ADSC-Exo group was highest compared with the control group and the ADSC-Exo group.

\section{Expressions of NLRP3 inflammasome after being incubated with S-ADSC-Exo}

ROS is an important activator of inflammasome. The effect of S-ADSC-Exo on ROS level was first explored showing that the ROS level in S-ADSC-Exo treated MAECs was significantly lower than that in the ADSC-Exo group and the control group (Fig. S3 a). Next, the qRT-PCR results of NLRP3 inflammasome were summarized in Fig. 3 a-c. The level of NLRP3 decreased slightly in the ADSC-Exo group. Transfection of STC-1 significantly decreased the level of NLRP3 in the S-ADSC-Exo group $(P<.05$, vs control). The level of Caspase-1 increased slightly in the ADSC-Exo group. Transfection of STC-1 significantly decreased the level of Caspase- 1 in the S-ADSC-Exo group $(P<.05$, vs control). The level of IL-1 $\beta$ decreased slightly in the ADSC-Exo group. Transfection of STC-1 significantly decreased the level of IL-1 $\beta$ in the S-ADSC-Exo group $(P<.05$, vs control).

Representative western blot pictures of NLRP3, Caspase-1, and IL-1 $\beta$ expressions were shown in Fig. $3 \mathrm{~d}$. Densitometric analysis revealed that transfection of STC-1 markedly decreased the expression of NLRP3 in the S-ADSC-Exo group (Fig. 3 e). There was no significant difference in the expression of NLRP3 between the control group and the ADSC-Exo group. Densitometric analysis revealed that transfection of STC-1 markedly decreased the expression of Caspase-1 in the S-ADSC-Exo group (Fig. $3 \mathrm{f}$ ). There was no significant difference in the expression of caspase-1 between the control group and the ADSC-Exo group. Densitometric analysis revealed that transfection of STC-1 markedly decreased the expression of IL-1 $\beta$ in the S-ADSC-Exo group (Fig. $3 \mathrm{~g}$ ). There was no significant difference in the expression of IL-1 $\beta$ between the control group and the ADSC-Exo group.

\section{Administration of siRNA-STC-1 reverses the reendothelialization of S-ASDC-Exo}

The scratch test and tube forming experiment were performed to assess the antagonist of siRNA targeting STC-1 on MAECs. Compared with the control group, excellent migration capacity was observed in the S-ADSC-Exo group (Fig. 4 a). However, administration of siRNA-STC-1 decreased the migration 
speed of S-ADSC-Exo. Blank area calculation showed that the siRNA-STC-1 group had the lowest migration speed at time intervals ( 6 h, 12 h, 24 h) (Fig. 4 c). The angiogenesis results revealed that tubeforming ability of MAECs was significantly inhibited in the group incubated with siRNA-STC-1. As shown in Fig. 4 b, d, tube density in the siRNA-STC-1 group was lowest compared with the S-ADSC-Exo group and the control group.

\section{Expressions of NLRP3 inflammasome after being incubated with siRNA-STC-1}

We used siRNA targeting STC-1 to further investigate the mechanism of S-ADSC-Exo promoting reendothelialization. The qRT-PCR results of STC-1 and NLRP3 inflammasome are summarized in Fig. 5 a-d. Transfection of siRNA targeting STC-1 significantly decreased the level of STC-1 in the siRNA-STC-1 group, but enhanced the levels of NLRP3, Caspase-1, and IL-1 $\beta$ in the siRNA-STC-1 group.

Representative western blot pictures of STC-1, NLRP3, Caspase-1, and IL-1 $\beta$ expressions are shown in Fig. 5 e. Densitometric analysis revealed that siRNA-STC-1 markedly decreased the expression of STC-1 (Fig. $5 \mathrm{f}$ ). Densitometric analysis revealed that siRNA-STC-1 markedly enhanced the expression of NLRP3 (Fig. $5 \mathrm{~g}$ ). Densitometric analysis revealed that siRNA-STC-1 markedly enhanced the expression of Caspase-1 (Fig. 5 h). Densitometric analysis revealed that siRNA-STC-1markedly enhanced the expression of IL-1 $\beta$ (Fig. 5 i).

\section{Expressions of NLRP3 inflammasome in the post-injury carotid arteries}

The effect of S-ADSC-Exo on ROS level was explored showing that the ROS level in S-ADSC-Exo treated carotid arteries was significantly lower than that in the ADSC-Exo group and that in the control group (Fig. S3 b). Representative western blot pictures of NLRP3, Caspase-1, and IL-1 $\beta$ are shown in Fig. 6 a. Densitometric analysis revealed that transfection of STC-1 markedly decreased the expression of NLRP3 in the S-ADSC-Exo group (Fig. 6 b). There was no significant difference in the expression of NLRP3 between the control group and the ADSC-Exo group. Densitometric analysis revealed that transfection of STC-1 markedly decreased the expression of Caspase-1 in the S-ADSC-Exo group (Fig. 6 c). There was no significant difference in the expression of Caspase-1 between the control group and the ADSC-Exo group. Densitometric analysis revealed that transfection of STC-1 markedly decreased the expression of IL-1 $\beta$ in the S-ADSC-Exo group (Fig. 6 d). There was no significant difference in the expression of IL-1 $\beta$ between the control group and the ADSC-Exo group.

\section{Immunofluorescence staining and histological examination of the post-injury carotid arteries}

Representative sections of endothelial cells stained with Ve-cad (red), NLRP3 inflammasome (green), and merge (orange) are shown in Fig. 7 a-c. As summarized in Fig. 7 a-c, the expressions of NLRP3, Caspase1 and IL-1 $\beta$ in the S-ADSC-Exo group were much lesser than that in the control group and that in the ADSC-Exo group. HE staining (Fig. $7 \mathrm{~d}$, e) showed that the thickness of arterial wall was much thinner in the S-ADSC-Exo group, compared with the control group and the ADSC-Exo group. Moreover, Even's blue 
staining (Fig. 7 d) demonstrated that the number of living endothelial cells was significantly greater than that in the control group and that in the ADSC-Exo group.

\section{Discussion}

The salient findings revealed by this current study are that the reendothelialization effect of S-ADSC-Exo

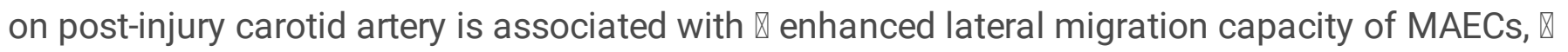

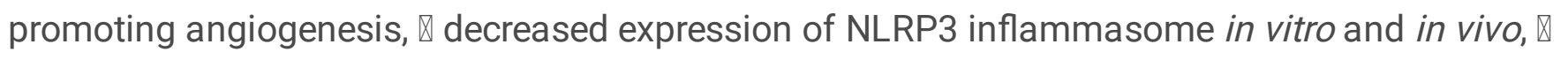
suppressed negative remodeling of arterial wall.

ADSCs are abundant in source, easy to harvest and isolate, can robustly release exosomes, and play a crucial role in tissue repair[25]. Collective data have indicated that exosomes released from ADSCs conduct vascular repair by promoting vascular plasticity[26], enhancing angiogenesis[26], improving post-injury vascular regeneration[27], and regulating autophagy[28]. We revealed that exosomes from genetically modified ADSCs induced reendothelialization effects on post-injury carotid endarterium as evidenced by improvement of carotid artery remodeling and survival of endothelial cells. Inflammatory response is consequence of mechanical injury using endovascular techniques and may be a principal contributor to complex inflammatory reactions of endothelial cells[29]. NLRP3 inflammasome are a group of intracellular protein complexes produced during inflammation activation, and act as innate immune signal receptor to initiate inflammatory response. IL $-1 \beta$, as an important member and a major executor of NLRP3 inflammasome, is strongly expressed in the chronic inflammation and restenosis after endovascular treatment[12, 19]. In the current study, the elevation of NLRP3 inflammasome (NLRP3, Caspase-1, and IL-1 $\beta$ ) after scratching was significantly inhibited by exosomes from ADSCs overexpressing STC-1, suggesting that exosomes may account for reendothelialization via antiinflammation effects. It is worth noting that compared with the control group, the lateral migration capacity and the tube density is higher in the ADSC-Exo group. On the contrary, the NLRP3 inflammasome in MAECs was enhanced after administration of siRNA targeting STC-1, and improvements of reendothelialization were abolished by the antagonist of siRNA-STC-1, indicating that NLRP3 inflammasome was involved in mediating reendothelialization of upregulation of STC-1. Although STC-1 was highlighted, some cargoes aside from STC-1 in ADSC-Exo may contribute to the reendothelialization and angiogenesis, which needs to be further investigated.

RNAs are important regulatory factors delivered by exosomes to target cells. Increasing reports indicates that ADSC-derived exosomes regulate target cell protein expression and cell morphological change. ADSC-derived exosomes promote wound-healing in diabetic mice through microRNA-128-3p/SIRT1 mechanism[28]. Via exosomes, ADSCs communicate with cardiomyocytes and macrophages to ameliorate ischemic injury by activating S1P/SK1/S1PR1 signaling pathway and foster M2 polarization[30]. Microglia-induced neural inflammatory injury can be suppressed by ADSC-exosomes through the NF-kB/MAPK pathway[31]. All these data show that the function ADSC-exosome can be enhanced by changing their RNA content. STC-1 is a conserved glycoprotein that can directly act on mitochondria, regulate oxidative phosphorylation and inhibit inflammatory reactions[23, 32]. By inhibiting 
oxidative stress, STC-1 restrain renal ischemia-reperfusion injury through ROS-mediated multiple signaling pathways[33]. Through being encapsulated within the exosomes, the STC-1 or other RNAs is protected from the degradation of protease or RNase[34]. Compared with un-transfected groups, transfection of STC-1 enhanced the levels of STC-1 in ADSCs, released exosomes, and MAECs engulfing exosomes. The content of ROS was decreased in carotid arteries after administration of S-ADSC-Exo. However, the same result occurred in the ADSC-Exo group, there may be some other active biomolecules within the exosomes played the alike role of STC-1, which needs to be further studied.

To elucidate the potential reendothelialization effect of S-ADSC-Exo on post-injury carotid endarterium, possibilities for the related proteins were explored. STC-1 is indicated to be an endogenous regulator of ROS[35], and ROS is the key signal to activate NLRP3 inflammasome[36]. ROS is a major mediator of the inflammation follows mechanical injury of endovascular treatment. Mechanical injury triggers an overproduction of ROS, which leads to complex change of intracellular protein expressions and results in cell apoptosis and pyroptosis[37]. ROS was reported to activate NLRP3 inflammasome by promoting the link of Thioredoxin-interacting protein (TXNIP) and NLRP3[38]. The expressions of NLRP3, Caspase-1, and IL-1 $\beta$ were detected to be activated in MAECs and in carotid arteries, indicating that the expressions of NLRP3 inflammasome were in consistent with the content of ROS and ROS was involved in the activation of NLRP3 inflammasome. Another important finding in this study was that STC-1 was transferred into carotid arteries by STC-1 enriched ADSC-exosomes, and the expressions of NLRP3 inflammasome were decreased as evidenced by immunofluorescence staining. In the meanwhile, the negative remodeling of arterial wall and the survival of endothelial cells were promoted. Therefore, it is plausible that STC-1 is mediator of NLRP3 inflammasome by which STC-1 enriched ADSC-exosomes attenuated the oxidative stress after mechanical injury of carotid artery and mediated reendothelialization effects on post-injury carotid endarterium compared with ADSC-derived exosomes.

\section{Conclusion}

Exosomes derived from genetically modified ADSCs conduct reendothelialization effect on post-injury carotid endarterium through STC-1/NLRP3 inflammasome pathway.

\section{Declarations}

\section{Ethical Approval}

The animal protocol was approved by the Ethics Review Committee of Animal Experimentation of Shanghai Jiao Tong University School of Medicine (Shanghai, People's Republic of China).

\section{Consent to Participate}

Not applicable

\section{Consent to Publish}


Not applicable

\section{Author's contributions}

$K L, H H S$, and $Z Y P$ contributed equally to this work. $X Y W, W M L$, and $X W L$ contributed equally to this work. K L, HH S, and ZY P contributed to experimental operation, data collection and analysis, manuscript writing. XY W and XW L contributed to model development, code development, and editing the manuscript. WM L contributed to manuscript proofreading.

\section{Funding}

This work was supported by the National Natural Science Foundation of China (Grant 81701801, 81971712, 81701842, and 81870346).

\section{Competing Interests}

The authors declare no conflicts of interest.

\section{Availability of data and materials}

The data that support the findings of this study are available on reasonable request from the corresponding author.

\section{References}

1. Zhu, Z., \& Yu, W. (2020). Update in the treatment of extracranial atherosclerotic disease for stroke prevention. Stroke and Vascular Neurology, 5, 65-70.

2. Saw, J. (2014). Carotid artery stenting for stroke prevention. Candian Journal of Cardiology, 30, 2234.

3. Wangqin, R., Krafft, P.R., Piper, K., Kumar, J., Xu, K., Mokin, M., et al. (2019). Management of De Novo Carotid Stenosis and Postintervention Restenosis-Carotid Endarterectomy Versus Carotid Artery Stenting-a Review of Literature. Translational Stroke Research, 10, 460-474.

4. Chaabane, C., Otsuka, F., Virmani, R., \& Bochaton-Piallat, M.L. (2013). Biological responses in stented arteries. Cardiovascular Research, 99, 353-363.

5. Wu, B., Mottola, G., Schaller, M., Upchurch, G.R., Jr., \& Conte, M.S. (2017). Resolution of vascular injury: Specialized lipid mediators and their evolving therapeutic implications. Molecular Aspects of Medcine, 58, 72-82.

6. Gotlieb, A.L., Spector, W., Wong, M.K., \& Lacey, C. (1984). In vitro reendothelialization. Microfilament bundle reorganization in migrating porcine endothelial cells. Arteriosclerosis, 4, 91-95.

7. Wang, Y., Liu, J., Ma, J., Sun, T., Zhou, Q., Wang, W., et al. (2019). Exosomal circRNAs: biogenesis, effect and application in human diseases. Molecular Cancer, 18, 116. 
8. Piffoux, M., Nicolas-Boluda, A., Mulens-Arias, V., Richard, S., Rahmi, G., Gazeau, F., et al. (2019).

Extracellular vesicles for personalized medicine: The input of physically triggered production, loading and theranostic properties. Advanced Drug Delivery Reviews, 138, 247-258.

9. Bouchareychas, L., Duong, P., Covarrubias, S., Alsop, E., Phu, T.A., Chung, A., et al. (2020).

Macrophage Exosomes Resolve Atherosclerosis by Regulating Hematopoiesis and Inflammation via MicroRNA Cargo. Cell Reports, 32, 107881.

10. Loyer, X., Zlatanova, I., Devue, C., Yin, M., Howangyin, K.Y., Klaihmon, P., et al. (2018). Intra-Cardiac Release of Extracellular Vesicles Shapes Inflammation Following Myocardial Infarction. Circulation Research, 123, 100-106.

11. Ma, T., Sun, J., Zhao, Z., Lei, W., Chen, Y., Wang, X., et al. (2017). A brief review: adipose-derived stem cells and their therapeutic potential in cardiovascular diseases. Stem Cell Research and Therapy, 8 , 124.

12. Cai, C., Kilari, S., Zhao, C., Simeon, M.L., Misra, A., Li, Y., et al. (2020). Therapeutic Effect of Adipose Derived Mesenchymal Stem Cell Transplantation in Reducing Restenosis in a Murine Angioplasty Model. Journal of the American Soceity of Nephrology, 31, 1781-1795.

13. Lin, Y., Zhu, W., \& Chen, X. (2020). The involving progress of MSCs based therapy in atherosclerosis. Stem Cell Research and Therapy, 11, 216.

14. Munoz, M.F., Arguelles, S., Guzman-Chozas, M., Guillen-Sanz, R., Franco, J.M., et al. (2018). Cell tracking, survival, and differentiation capacity of adipose-derived stem cells after engraftment in rat tissue. Journal of Cellular Physiology, 233, 6317-6328.

15. Yan, W., \& Jiang, S. (2020). Immune Cell-Derived Exosomes in the Cancer-Immunity Cycle. Trends in Cancer, 6, 506-517.

16. Ko, K.W., Yoo, Y.I., Kim, J.Y., Choi, B., Park, S.B., Park, W., et al. (2020). Attenuation of Tumor Necrosis Factor-alpha Induced Inflammation by Umbilical Cord-Mesenchymal Stem Cell Derived ExosomeMimetic Nanovesicles in Endothelial Cells. Tissue Engineering and Regenerative Medicine, 17, 155163.

17. Fan, B., Li, C., Szalad, A., Wang, L., Pan, W., Zhang, R., et al. (2020). Mesenchymal stromal cell-derived exosomes ameliorate peripheral neuropathy in a mouse model of diabetes. Diabetologia, 63, 431443.

18. Ye, Y., Zhang, X., Xie, F., Xu, B., Xie, P., Yang, T., et al. (2020). An engineered exosome for delivering sgRNA:Cas9 ribonucleoprotein complex and genome editing in recipient cells. Biomaterials Science, 8, 2966-2976.

19. Wei, W., Li, X.X., \& Xu, M. (2019). Inhibition of vascular neointima hyperplasia by FGF21 associated with FGFR1/Syk/NLRP3 inflammasome pathway in diabetic mice. Atherosclerosis, 289, 132-142.

20. Swanson, K.V., Deng, M., \& Ting, J.P. (2019). The NLRP3 inflammasome: molecular activation and regulation to therapeutics. Nature Reviews Immunology, 19, 477-489.

21. Sorbara, M.T., \& Girardin, S.E. (2011). Mitochondrial ROS fuel the inflammasome. Cell Research, 21, 558-560. 
22. Li, X., Zhou, H., Guo, D., Hu, Y., Fang, X., Chen, Y., et al. (2020). Oxidative stress and inflammation: Early predictive indicators of multiple recurrent coronary in-stent chronic total occlusions in elderly patients after coronary stenting. IUBMB Life, 72, 1023-1033.

23. Oh, J.Y., Ko, J.H., Lee, H.J., Yu, J.M., Choi, H., Kim, M.K., et al. (2014). Mesenchymal stem/stromal cells inhibit the NLRP3 inflammasome by decreasing mitochondrial reactive oxygen species. Stem Cells, 32, 1553-1563.

24. Wang, Y., Chu, Y., Li, K., Zhang, G., Guo, Z., Wu, X., et al. (2020). Exosomes Secreted by AdiposeDerived Mesenchymal Stem Cells Foster Metastasis and Osteosarcoma Proliferation by Increasing COLGALT2 Expression. Frontiers in Cell and Developmental Biology, 8, 353.

25. Cai, J., Wu, J., Wang, J., Li, Y., Hu, X., Luo, S., et al. (2020). Extracellular vesicles derived from different sources of mesenchymal stem cells: therapeutic effects and translational potential. Cell and Bioscience, 10, 69.

26. Hoang, D.H., Nguyen, T.D., Nguyen, H.P., Nguyen, X.H., Do, P.T.X., Dang, V.D., et al. (2020). Differential Wound Healing Capacity of Mesenchymal Stem Cell-Derived Exosomes Originated From Bone Marrow, Adipose Tissue and Umbilical Cord Under Serum- and Xeno-Free Condition. Frontiers in Molecular Biosciences, 7, 119.

27. Li, X., Xie, X., Lian, W., Shi, R., Han, S., Zhang, H., et al. (2018). Exosomes from adipose-derived stem cells overexpressing Nrf2 accelerate cutaneous wound healing by promoting vascularization in a diabetic foot ulcer rat model. Experimental and Molecular Medicine, 50, 29.

28. Shi, R., Jin, Y., Hu, W., Lian, W., Cao, C., Han, S., et al. (2020). Exosomes derived from mmu_circ_0000250-modified adipose-derived mesenchymal stem cells promote wound healing in diabetic mice by inducing miR-128-3p/SIRT1-mediated autophagy. American Journal of PhysiologyCell Physiology, 318, C848-C856.

29. Davis, C., Fischer, J., Ley, K., \& Sarembock, I.J. (2003). The role of inflammation in vascular injury and repair. Journal of Thrombosis and Haemostasis, 1, 1699-1709.

30. Deng, S., Zhou, X., Ge, Z., Song, Y., Wang, H., Liu, X., et al. (2019). Exosomes from adipose-derived mesenchymal stem cells ameliorate cardiac damage after myocardial infarction by activating S1P/SK1/S1PR1 signaling and promoting macrophage M2 polarization. International Journal of Biochemstry \& Cell Biology, 114, 105564.

31. Feng, N., Jia, Y., \& Huang, X. (2019). Exosomes from adipose-derived stem cells alleviate neural injury caused by microglia activation via suppressing NF-kB and MAPK pathway. Journal of Neuroimmunology, 334, 576996.

32. Sheikh-Hamad, D. (2010). Mammalian stanniocalcin-1 activates mitochondrial antioxidant pathways: new paradigms for regulation of macrophages and endothelium. American Journal of Physiology-Renal Physiology, 298, F248-254.

33. Liu, D., Shang, H., \& Liu, Y. (2016). Stanniocalcin-1 Protects a Mouse Model from Renal IschemiaReperfusion Injury by Affecting ROS-Mediated Multiple Signaling Pathways. International Journal of Molecular Sciences, 17, 1051. 
34. Valadi, H., Ekstrom, K., Bossios, A., Sjostrand, M., Lee, J.J., \& Lotvall, J.O. (2007). Exosome-mediated transfer of mRNAs and microRNAs is a novel mechanism of genetic exchange between cells. Nature Cell Biology, 9, 654-659.

35. Yang, K., Yang, Y., Qi, C., \& Ju, H. (2020). Effects of porcine STC-1 on cell metabolism and mitochondrial function. Genernal and Comparative Endocrinology, 286, 113298.

36. Tschopp, J., \& Schroder, K. (2010). NLRP3 inflammasome activation: The convergence of multiple signalling pathways on ROS production? Nature Reviews Immunology, 10, 210-215.

37. Robinson, N., Ganesan, R., Hegedus, C., Kovacs, K., Kufer, T.A., \& Virag, L. (2019). Programmed necrotic cell death of macrophages: Focus on pyroptosis, necroptosis, and parthanatos. Redox Biology, 26, 101239.

38. Choe, J.Y., \& Kim, S.K. (2017). Quercetin and Ascorbic Acid Suppress Fructose-Induced NLRP3 Inflammasome Activation by Blocking Intracellular Shuttling of TXNIP in Human Macrophage Cell Lines. Inflammation, 40, 980-994.

\section{Figures}



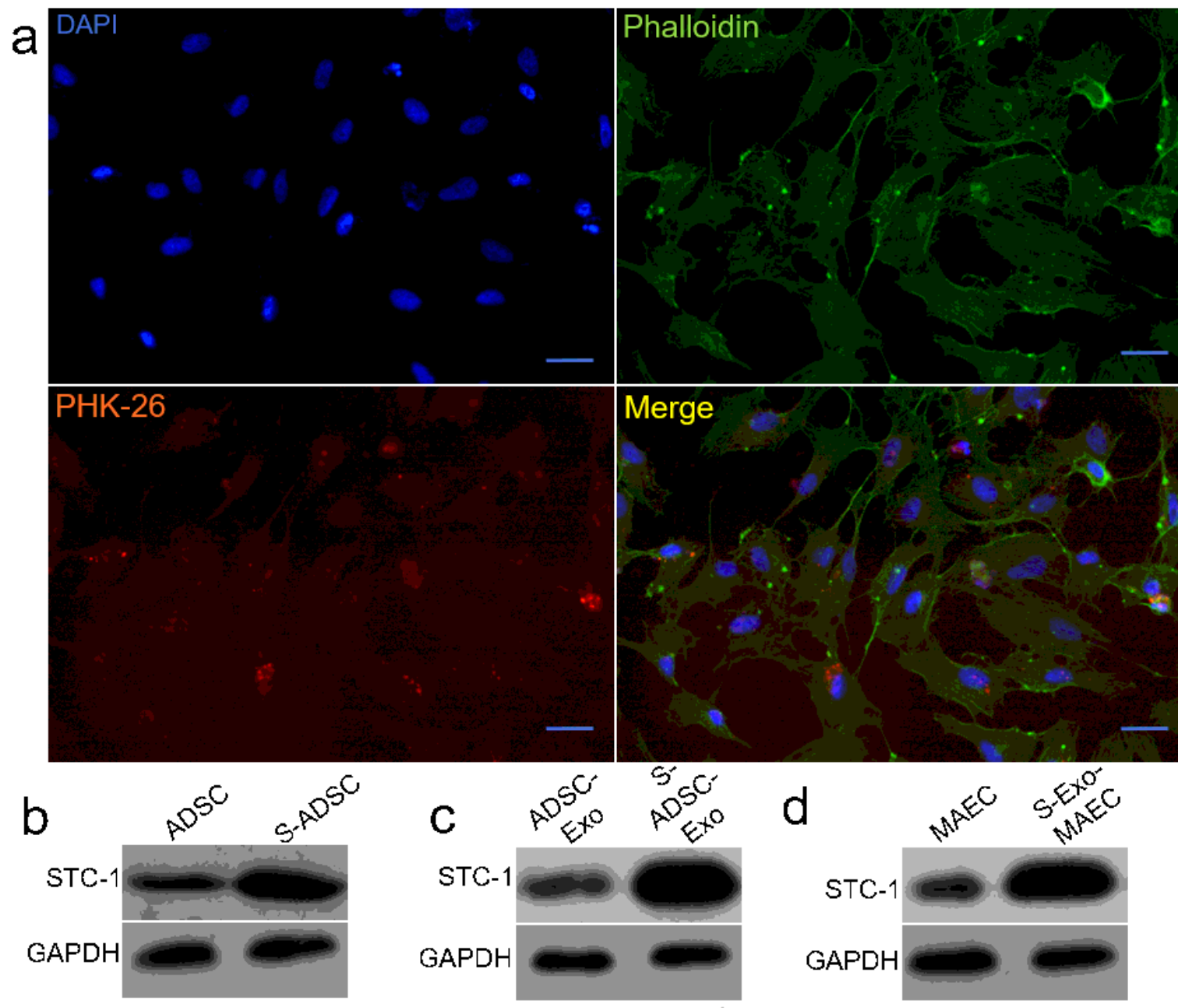

e

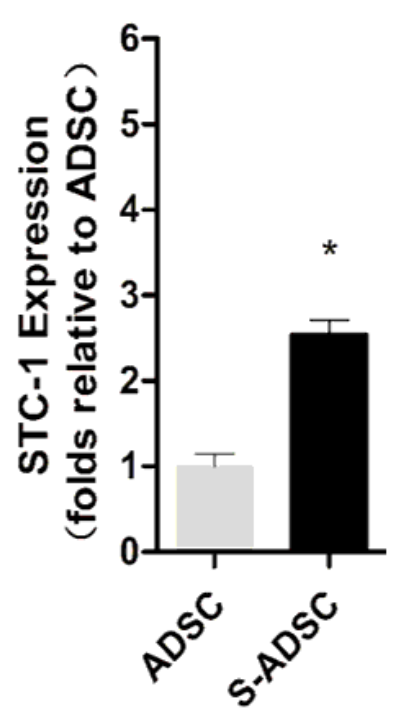

f

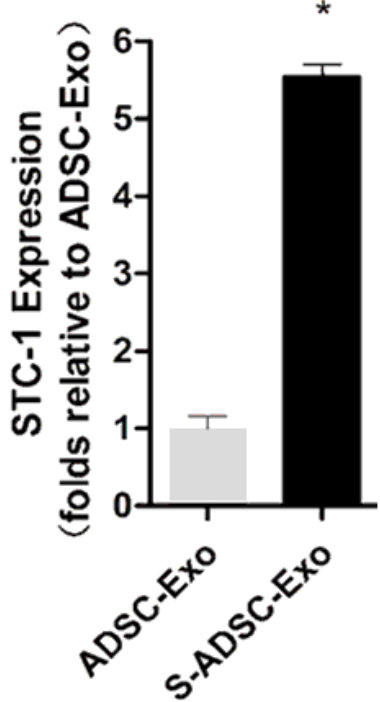

g

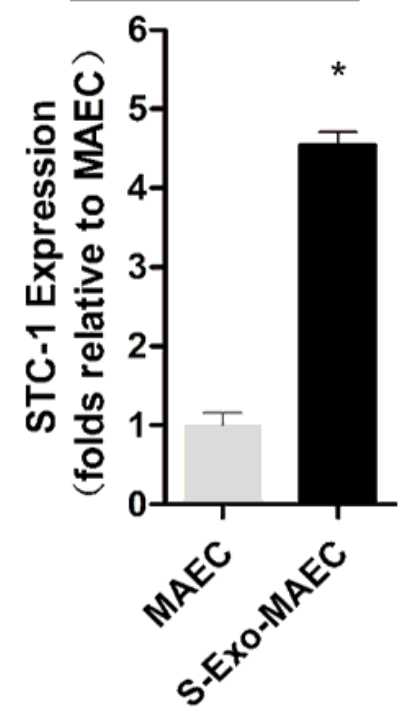

\section{Figure 1}

ADSC-Exosomes engulfed by MAECs and expressions of STC-1 after transfection. a Representative image of PHK-26 labelled ADSC-Exo (red) and Phalloidin (green) double-immunostaining in MAECs. Nuclei were stained with DAPI (blue). b-d Representative western blot pictures showing STC-1 expression. e Densitometric quantification of STC-1 expression in S-ADSC group, vs un-transfected. $f$ Densitometric quantification of STC-1 expression in S-ADSC-Exo group, vs un-transfected. g Densitometric 
quantification of STC-1 expression in S-Exo-MAEC group, vs un-transfected. * PQ .05, compared with untransfected groups. S-ADSC, ADSCs transfected with STC-1; S-Exo-MAEC, MAEC engulfing S-ADSC-Exo. Scale bar $=200 \square \mathrm{m}$.

\section{Figure 2}

Reendothelialization effect of S-ADSC-Exo in vitro. a Representative pictures showing lateral migration capacity at the time intervals (6h, $12 \mathrm{~h}$, and $24 \mathrm{~h}$ ). b Representative pictures showing tube forming. $\mathrm{c}$ Calculation of blank area according to figure $a$. $d$ Calculation of tube density according to figure $b .{ }^{*}[$ .05, vs control; \#PQ.05, S-ADSC-Exo vs ADSC-Exo; \$ PQ.05, Control vs ADSC-Exo. Scale bar $=200 \square \mathrm{m}$.

\section{Figure 3}

Effects of S-ADSC-Exo administration on expressions of NLRP3 inflammasome in vivo. a-c mRNA levels of NLRP3, caspase-1, and IL-1 $\beta$. $d$ Representative western blot pictures showing expressions of NLRP3, Caspase-1, and IL-1 $\beta$. e-g Densitometric quantification of NLRP3, Caspase-1, and IL-1 $\beta$ expression. *PI .05 , vs control. EC, endothelial cell.

\section{Figure 4}

Effects of siRNA-STC-1 on reendothelialization. a Representative pictures showing lateral migration capacity at the time intervals ( $6 \mathrm{~h}, 12 \mathrm{~h}$, and $24 \mathrm{~h})$. b Representative pictures showing tube forming. $\mathrm{c}$ Calculation of blank area according to figure $a$. $d$ Calculation of tube density according to figure $b . * P[$ .05, vs control; \#PQ .05, S-ADSC-Exo vs siRNA-STC-1; \$ PQ .05, S-ADSC-Exo vs control. Scale bar $=200 \square \mathrm{m}$.

\section{Figure 5}

Effects of siRNA-STC-1 administration on expressions of NLRP3 inflammasome in vitro. a-b mRNA levels of NLRP3, caspase-1, and IL-1 $\beta$. e Representative western blot pictures showing expressions of STC-1, NLRP3, Caspase-1, and IL-1 $\beta$. f-i Densitometric quantification of STC-1, NLRP3, Caspase-1, and IL-1 $\beta$ expression. *PQ .05, vs control; \# PQ .05, siRNA-STC-1 vs S-ADSC-Exo.

\section{Figure 6}

Effects of S-ADSC-Exo administration on expressions of NLRP3 inflammasome in vivo. a Representative western blot pictures showing expressions of NLRP3, Caspase-1, and IL-1 $\beta$. b-d Densitometric quantification of NLRP3, caspase-1, and IL-1 $\beta$ expressions. *P区.05, vs control. 

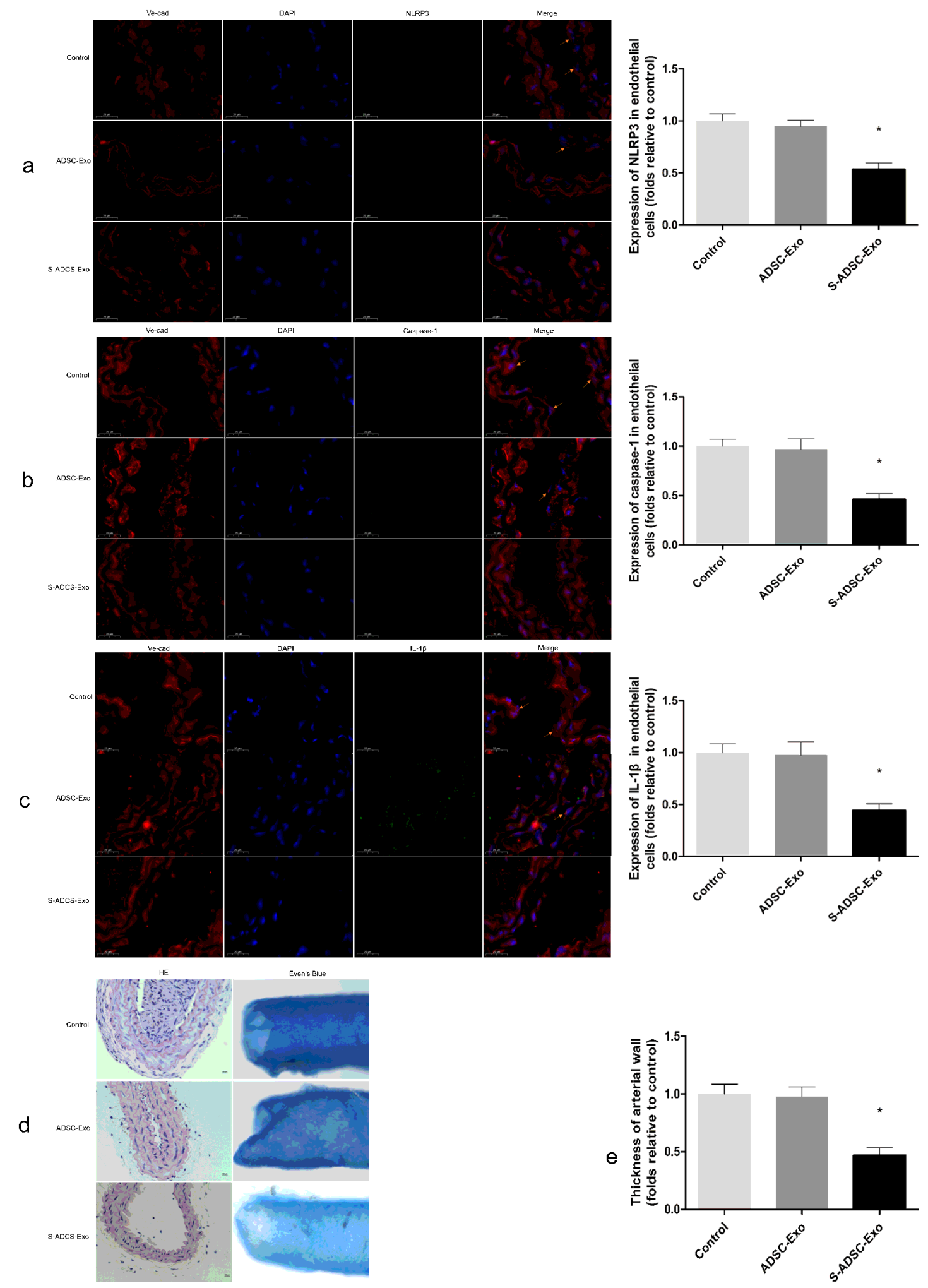

Figure 7

Immunofluorescence staining and HE staining of carotid arteries. a Representative images of IF staining and quantification of NLRP3 expression. b Representative images of IF staining and quantification of Caspase-1 expression. c Representative images of IF staining and quantification of IL-1 $\beta$ expression. $d$ Representative images of HE staining and Even's blue staining. e Quantification of thickness of arterial 
wall. Yellow arrows indicating endothelial cells. HE, hematoxylin-eosin. ${ }^{*} \mathrm{P} \otimes .05$, vs control. IF scale bar $=$ $20 \rrbracket \mathrm{m} ; \mathrm{HE}$ scale bar $=1000 \mathrm{~m}$.

\section{Supplementary Files}

This is a list of supplementary files associated with this preprint. Click to download.

- Supplement.docx

- Figs1.tif

- Figs2.tif

- Figs3.tif 